\title{
CXCR1/Akt signaling activation induced by mesenchymal stem cell-derived IL-8 promotes osteosarcoma cell anoikis resistance and pulmonary metastasis
}

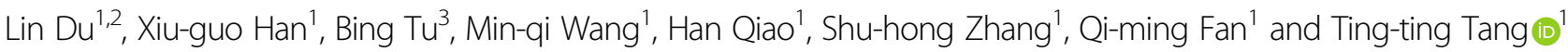

\begin{abstract}
The loss of appropriate cell adhesion normally induces apoptosis via a process termed anoikis. The aim of this study was to investigate the effects of mesenchymal stem cells (MSCs) in the cancer microenvironment on the anoikis resistance and pulmonary metastasis of osteosarcoma (OS) cells, and to evaluate the critical role of the interleukin (IL)8/C-X-C chemokine receptor (CXCR) 1/Akt-signaling pathway in these processes. Metastatic OS subtype cells, which did or did not interact with MSC-conditioned medium (MSC-CM) in vitro, were isolated from the pulmonary site and named Saos2-lung-M. Both MSC-CM and IL-8 treatment increased the anoikis resistance of Saos2 cells in vitro. Moreover, exogenous MSC-CM promoted the survival and metastasis of Saos2 cells in nude mice. Saos2-lung-M cells were more malignant and resistant to anoikis than parental cells. MSCs secreted IL-8, thereby protecting OS cells from anoikis. Blocking the IL-8/CXCR1/Akt pathway via CXCR1 knockdown inhibited the pulmonary metastasis of Saos2lung-MSCs and prolonged the survival of tumor-bearing mice. In conclusion, MSCs enhanced OS cell resistance to anoikis and pulmonary metastasis via regulation of the IL-8/CXCR1/Akt pathway. These findings suggest that MSCs can "select for" OS cells with high metastatic potential in vivo, and highlight CXCR1 as a key target in the regulation of pulmonary metastasis of OS cells.
\end{abstract}

\section{Introduction}

Osteosarcoma (OS) is a common primary malignancy of the bone, typically occurring in children and adolescents with an age-standardized incidence of approximately five per million cases per year ${ }^{1-3}$. OS originates from primitive mesenchymal bone-forming cells and often occurs in the long bones such as the proximal tibia and distal femur ${ }^{4,5}$. Current OS treatment regimens consist of a combination of surgery and intensive multi-agent chemotherapy, and

\footnotetext{
Correspondence: T-t. Tang (ttt@sjtu.edu.cn) or Q-m. Fan (chillow@163.com) ${ }^{1}$ Shanghai Key Laboratory of Orthopedic Implants, Department of Orthopedic Surgery, Shanghai Ninth People's Hospital, Shanghai Jiao Tong University School of Medicine, Shanghai, China

2Department of Orthopaedic Surgery, Renji Hospital, Shanghai Jiao Tong University School of Medicine, Shanghai, China

Full list of author information is available at the end of the article.

These authors contributed equally: Lin Du, Xiu-guo Han.
}

overall survival rates for non-metastatic OS are approximately $50-70 \%{ }^{6}$. However, $30-40 \%$ of patients with OS experience pulmonary metastasis and relapse, which are associated with significantly poor prognoses; indeed, the overall 5 -year survival rate is only about $20 \%^{7}$. Amputation of the affected limbs is often the only remaining treatment option; however, even this intervention usually fails to save patient lives due to early metastases ${ }^{8}$. Therefore, the development of novel techniques for preventing and treating OS metastases is highly desired.

As a barrier to metastasis, cells normally undergo apoptosis upon losing contact with their associated extracellular matrix or neighboring cells. This type of cell death is termed "anoikis" anoikis can survive longer when unattached; therefore, these cells are involved in cell migration and tissue 


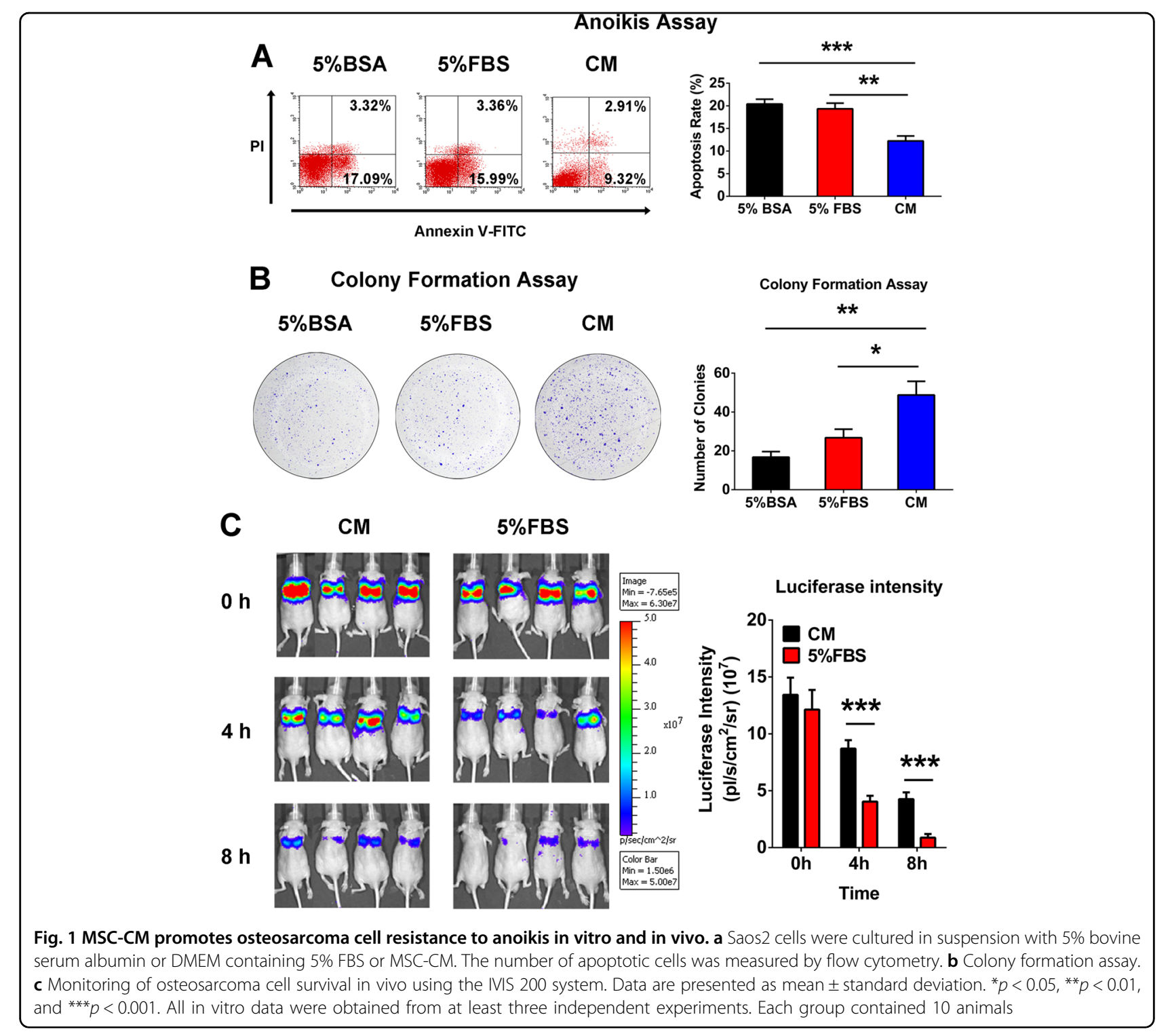

remodeling ${ }^{11}$. In addition, several studies have delineated the complex interactions between bone marrow-derived mesenchymal stem cells (MSCs) and tumor cells ${ }^{12,13}$. Metaphyses are the sites of OS predilection and are rich in MSCs; ${ }^{14}$ these conditions facilitate the interactions between MSCs and OS cells. Previous studies, including our own work ${ }^{15-17}$, have indicated that MSCs could promote tumor engraftment and metastatic colonization in a rat OS model. However, the role of MSCs in the OS resistance to anoikis and the underlying associated molecular mechanisms remain unknown.

MSCs exert a proinflammatory influence by constitutively secreting cytokines into the bone marrow microenvironment ${ }^{18}, 19$. Interleukin (IL)-8 is one of the predominant transcriptional targets of the inflammatory signaling mediated by nuclear factor- $\mathrm{kB}$, which is commonly activated in tumor microenvironments ${ }^{20}$. Migratory inhibitory factor-induced stromal protein kinase C $\beta / \mathrm{IL}-8$ is essential in human acute myeloid leukemia, and introduction of targeted IL-8 small hairpin ribonucleic acid (RNA) inhibits MSC-induced acute myeloid leukemia ${ }^{21}$. Furthermore, Avnet et al. ${ }^{22}$ suggested that the OS microenvironment is a key factor in MSC activation, which promotes the secretion of paracrine factors, such as IL-8 and IL-6 that significantly influence tumor behavior. Jiang et $\mathrm{al}^{23}$ showed that IL-8 promotes human OS cell invasion by regulating the phosphoinositide 3-kinase (PI3K)/protein kinase B (Akt) signaling pathway. Our previous studies also showed that expression of the IL-8-specific receptor $\mathrm{C}-\mathrm{X}-\mathrm{C}$ chemokine receptor (CXCR) 1 is upregulated in the highly metastatic OS cell line Saos2-lung ${ }^{24}$, and knockdown of CXCR1, which is regulated by IL-8/ CXCR1/Akt signaling, increased the sensitivity of the Saos2-lung cells to cisplatin. ${ }^{25}$. 


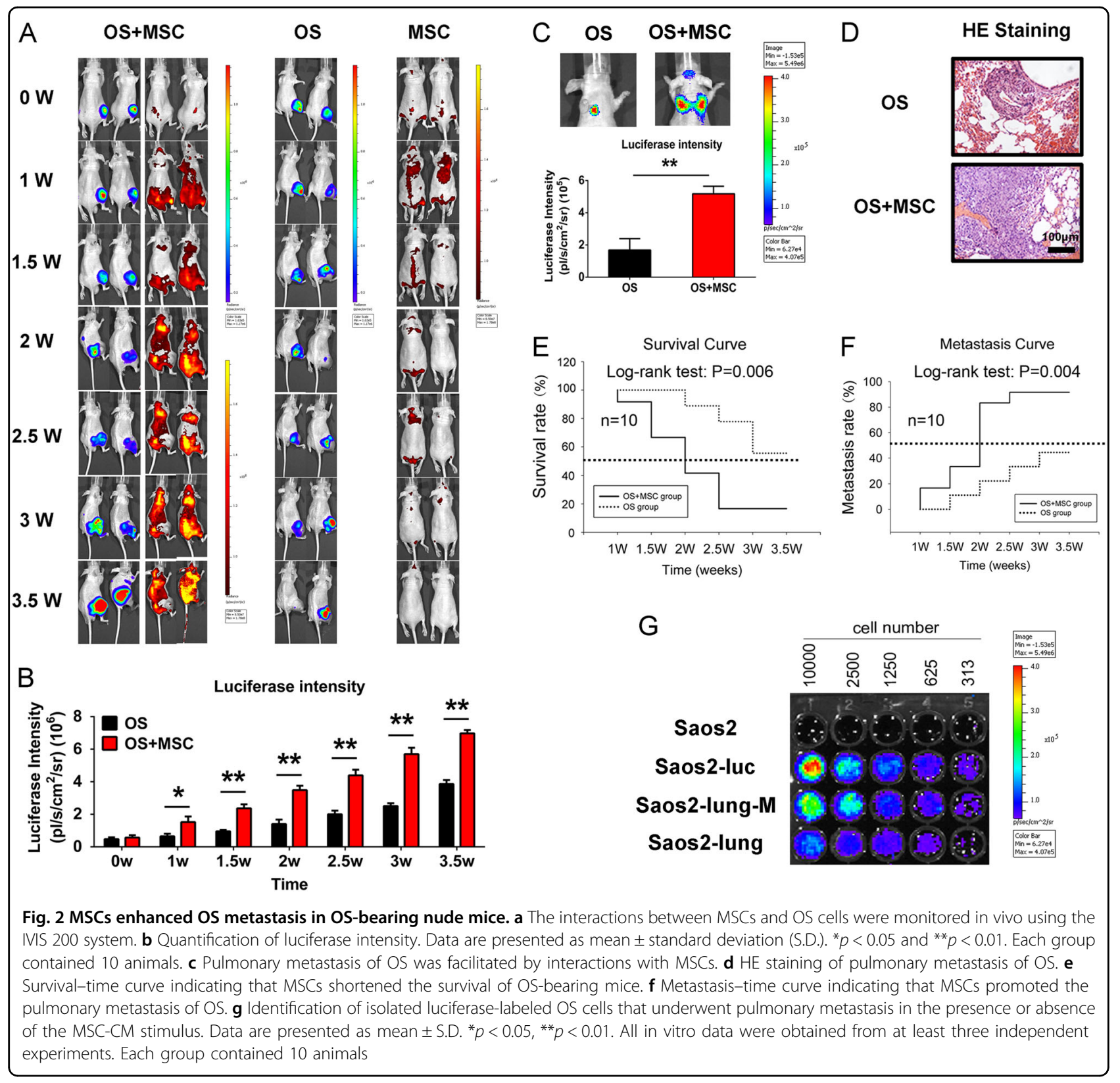

In the present study, we investigated the interactions between MSCs and OS cells using a living cell-tracing imaging system, and examined the influence of MSCs in the cancer microenvironment on OS cell anoikis resistance and pulmonary metastasis. Moreover, through isolation of highly metastatic OS cells, we determined an important role for the IL-8/CXCR1/Akt-signaling pathway in anoikis resistance.

\section{Results}

MSC-conditioned medium (CM) protected OS cells from anoikis in vitro and in vivo

To evaluate the effect of the MSC-CM on anoikis, Saos2 cells were cultured in suspension and anoikis was quantified based on the number of apoptotic cells as measured by flow cytometry. The results showed a lower rate of apoptosis in the cell population cultured in MSC$\mathrm{CM}$ than in the two control groups (5\% bovine serum albumin or $5 \%$ fetal bovine serum (FBS) group) (Fig. 1a). Next, 600 cells from the same groups were cultured in an adhesive state with the original stimulus to test their ability to form colonies. There were significantly more colonies detected from the MSC-CM-stimulated cells than control cells (Fig. 1b). Furthermore, we analyzed MSC-CM-treated and untreated Saos2 cells in vivo by monitoring OS cell survival in mice with an in vivo imaging system (IVIS). Similar to the in vitro results, we discovered that the MSC-CM-treated cells survived much 


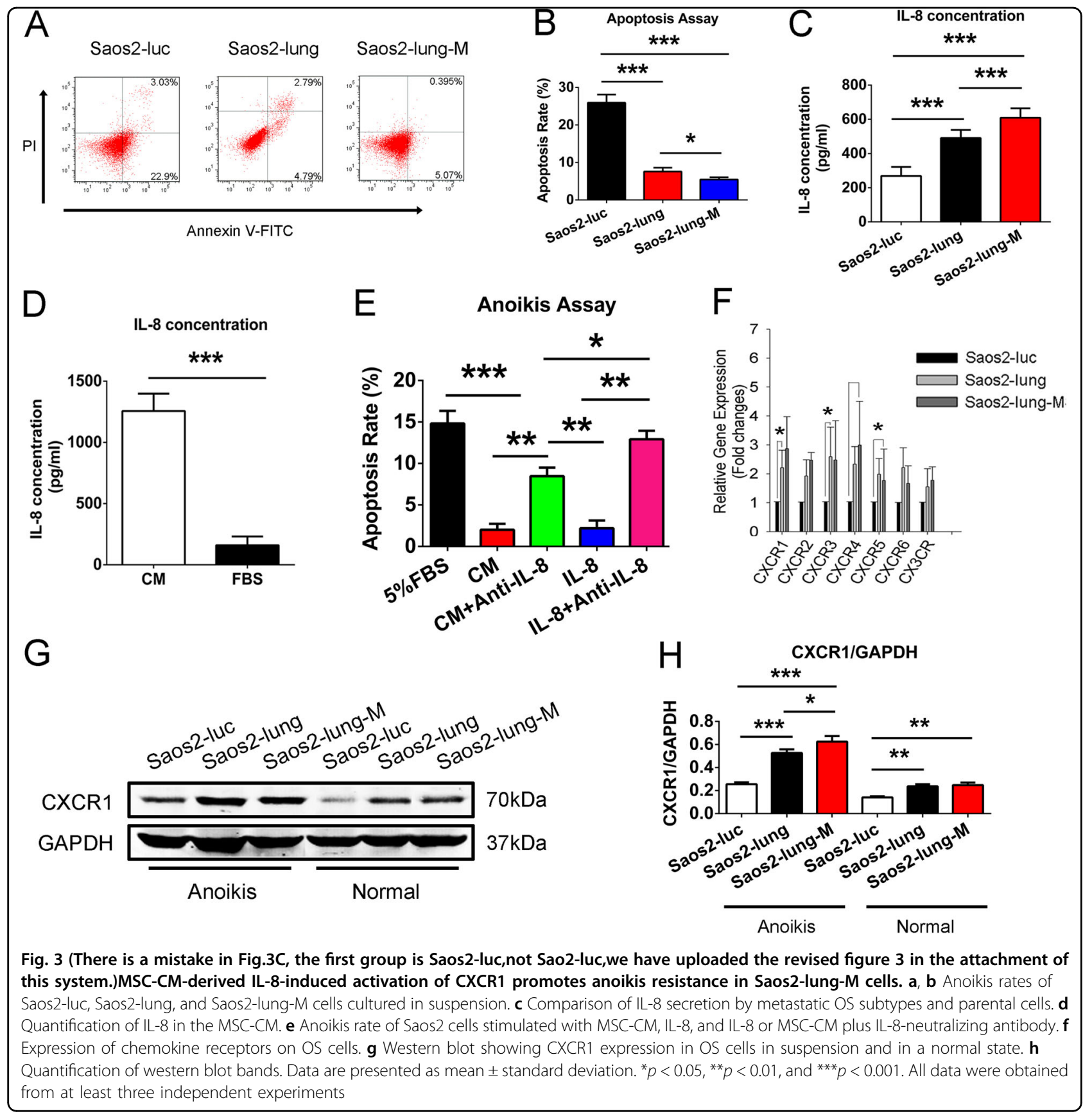

longer than the control cells (Fig. 1c). Therefore, we concluded that MSC-CM protected the OS cells from anoikis in vitro and promoted the survival of tumor cells in vivo.

The distribution of MSCs in OS-bearing nude mice and MSC enhancement of OS growth and metastasis

We established a primary OS model to verify the effect of MSCs on the growth and metastasis of Saos2 cells. After tumor formation for 4 weeks, red fluorescent protein (RFP)-labeled MSCs were injected via the tail vein into the OS model and control mice. An IVIS was used to monitor the growth of MSCs and OS cells for 3.5 weeks at half-week intervals. Figure $2 \mathrm{a}$ indicates that the MSCs targeted the OS with enhanced systemic distributions in OS-bearing nude mice. Quantitative data revealed that the MSCs notably promoted OS growth (Fig. 2b). Furthermore, the MSCs significantly enhanced OS metastasis (Fig. 2c). Hematoxylin and eosin (HE) staining confirmed that OS metastasis was severe in MSC-CM-treated cells (Fig. 2d). Survival and metastasis curves indicated that the MSCs facilitated pulmonary metastasis in OS-bearing 
nude mice and shortened their survival period (Fig. 2e, f). Next, luciferase-labeled Saos2-lung-M cells were isolated from the sites of pulmonary metastasis and identified in vitro, using Saos2-lung ${ }^{25}$ and Saos2-luc ${ }^{26}$ cells as positive controls and unlabeled Saos2 cells as the negative control (Fig. 2g). Thus, we monitored the MSC interactions with OS cells in real-time in vivo in a non-invasive manner and isolated Saos2-lung-M cells, which represent a subtype of metastatic OS cells after MSC treatment.

\section{Saos2-lung-M cells with IL-8/CXCR1 activation exhibited higher anoikis resistance}

When the number of cells undergoing anoikis was compared among Saos2-luc, Saos2-lung, and Saos2-lung$M$ cells, we found that fewer Saos2-lung-M cells underwent anoikis than Saos2-luc and Saos2-lung cells (Fig. 3a, b). Furthermore, enzyme-linked immunosorbent assay (ELISA) results show that Saos2-lung-M cells secreted more IL-8 than Saos2-luc and Saos2-lung cells (Fig. 3c), and that the MSC-CM contained more IL-8 than FBS (Fig. 3d). Further analysis revealed that both the MSC-CM and IL-8 protected OS cells from anoikis, and that IL-8neutralizing antibody blocked this effect (Fig. 3e). Moreover, the results of polymerase chain reaction (PCR) indicated that CXCR1, an IL-8-specific receptor, was most highly expressed in Saos2-lung-M cells (Fig. 3f). Western blot analysis confirmed that the Saos-lung and Saos2lung-M cells expressed more CXCR1 than Saos-luc cells at the protein level, both when in suspension and in a normal culture state (Fig. 3g, h). In conclusion, Saos2lung-M cells showed higher CXCR1 activation./CXCR1 activation.

\section{Akt signaling acts downstream of the IL-8/CXCR1 axis in OS cell anoikis}

The MSC-CM- and IL-8-stimulated phosphorylation of Akt protein was induced in suspended Saos 2 cells; however, IL-8-neutralizing antibody could block the phosphorylation of Akt (Fig. 4a). Immunofluorescence staining of IL-8-stimulated Saos2-luc, Saos2-lung, and Saos2-lung$M$ cells revealed that CXCR1 and p-Akt expression was more strongly upregulated in Saos2-lung-M cells than in Saos2-luc and Saos2-lung cells (Fig. 4b). Analysis of p-Akt protein levels in cells in suspension yielded parallel results (Fig. 4c). Therefore, the Akt-signaling pathway was activated in suspended OS cells by IL-8 in an anoikisinducing environment.

\section{CXCR1 knockdown inhibited OS growth and metastasis in vivo}

Knockdown of CXCR1 expression was performed in Saos2-lung-M cells (Fig. 5a), and the successful downregulation of CXCR1 expression was confirmed by realtime PCR (Fig. 5b). CXCR1 protein expression, as well as
p-Akt protein expression, also decreased (Fig. 5c). We then established a primary OS model and introduced CXCR1 knockdown or scrambled control Saos2-lung-M cells with RFP-labeled MSCs by intramedullary tibia injection. Optical imaging in vivo revealed that CXCR1 knockdown restrained OS growth and metastasis in mice, and the MSCs in the CXCR1-knockdown Saos2-lung-Mbearing nude mice completely disappeared at week 9 , while the scrambled control cells were maintained for 18 weeks (Fig. 5 d-f). Survival curves further indicated that CXCR1 knockdown prolonged the survival of OS-bearing nude mice (Fig. 5g), and pulmonary metastasis curves obtained using optical imaging indicated that CXCR1 knockdown suppressed lung metastasis (Fig. 5h). Accordingly, the tumor volume was smaller in the CXCR1 knockdown group than in the control (Fig. 5i, j). Therefore, we concluded that CXCR1 knockdown inhibited OS cell growth and metastasis and blocked the interactions between MSCs and OS cells in vivo.

\section{CXCR1 knockdown shortened the survival of OS cells in vivo and resulted in downregulation of CXCR1 and p-Akt expression in tumor samples}

We analyzed the survival of CXCR1-knockdown and scrambled control Saos2-lung-M cells in vivo and found that the CXCR1-knockdown cells survived for shorter periods than the control cells (Fig. 6a, b). Metastasis tumor tissues collected as of 4 weeks post-injection of CXCR1-knockdown and scrambled control Saos2-lung-M cells were immunostained for CXCR1 and p-Akt. HE staining revealed smaller metastatic nodes, and immunostaining showed reduced expression of CXCR1 and pAkt in the CXCR1-knockdown mice compared to the control mice (Fig. 6c, d). These data suggest that CXCR1 knockdown significantly reduced the tumor size and metastasis rate, as well as decreased the expression of CXCR1/Akt (Fig. 6e).

\section{Discussion}

OS is a primary malignant tumor, and has the highest incidence of all tumors treated in the department of orthopedics, characterized by a high rate of metastasis ${ }^{27}$. Anoikis is regarded as one of the mechanisms regulating metastasis, and is thus expected to be closely related to OS metastasis as well. Besides the OS cells themselves, the OS tumor microenvironment may have a strong relationship with anoikis regulation. We have observed that the predilection site of OS is also rich in bone marrow MSCs, and a previous study revealed that complex interactions occur between MSCs and tumor cells ${ }^{28}$. Although MSCs are known to play an important role in promoting tumor metastasis ${ }^{29}$, it is still unresolved as to whether MSCs can promote tumor cell resistance to anoikis and the colonization of distant tissues. Based on 


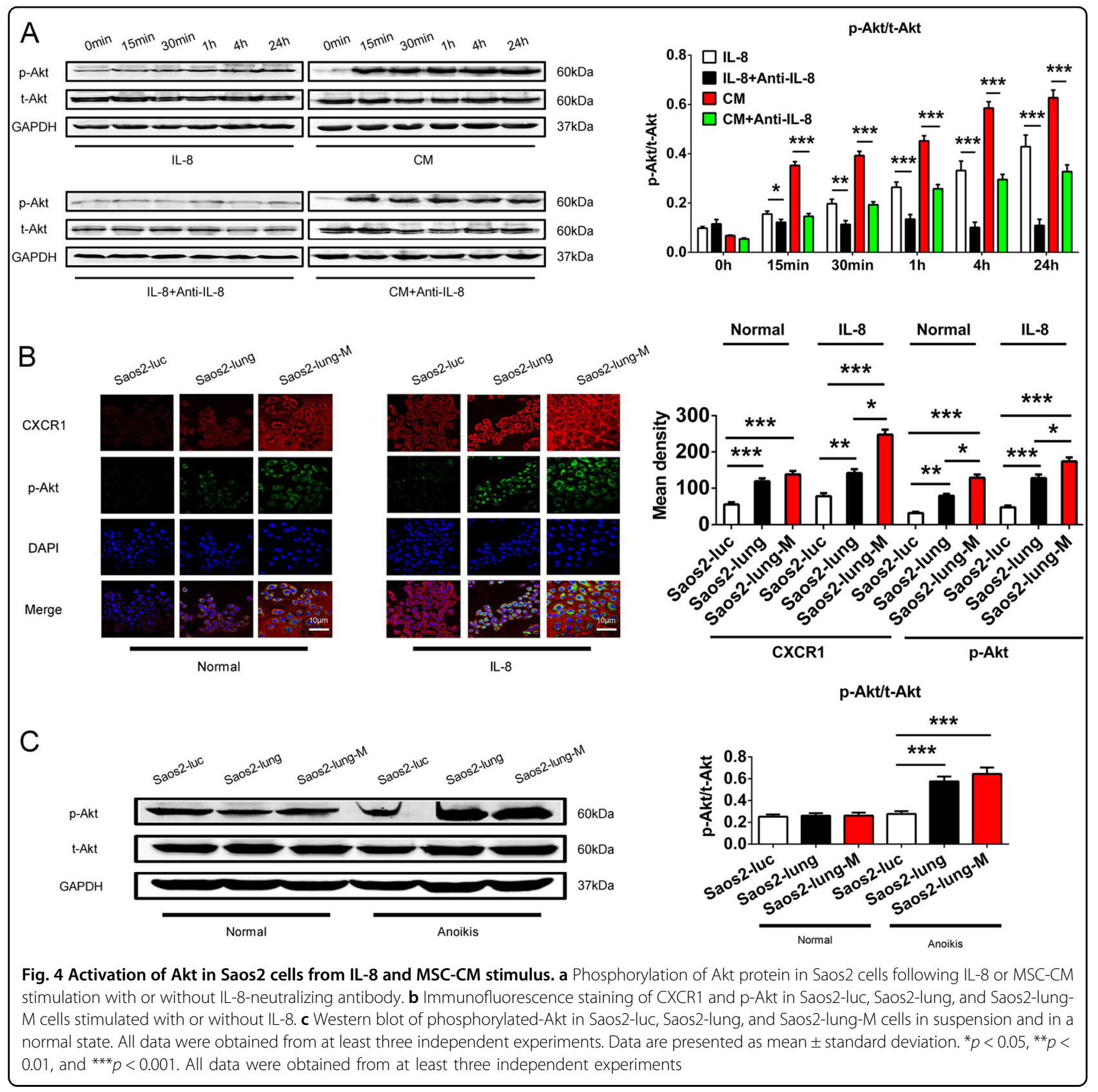

the specific characteristic of OS, we believe that OS is a good disease model with which to explore whether bone marrow MSCs can facilitate tumor cell metastasis by regulating anoikis.

In the present study, we confirmed that the active components in the MSC-CM inhibited OS cell anoikis, including IL-8. By analyzing the chemokine receptor family genes in metastatic OS cell subtypes obtained during our study, we found that the chemokine receptor CXCR1 is more highly expressed in Saos2-lung-M and Saos2-lung cells than in parental OS cells. However, many chemokine receptor ligands are not unique to the chemokine receptor family; ${ }^{30}$ that is, these chemokine receptors are not highly specific and can be activated by more than one component. CXCR1 has only been shown to have two ligands, IL- 8 and C-X-C motif chemokine ligand (CXCL)6, with high affinity only for IL-8 interactions ${ }^{31}$.

It is crucial to further elaborate the mechanisms driving this phenomenon. As a key protein in the regulation of cell survival, Akt protein plays an important role in anoikis $^{32}$. Phosphorylation of Akt protein can produce a series of biological effects ${ }^{33}$. Previous studies have demonstrated a relationship between IL-8 and Akt 


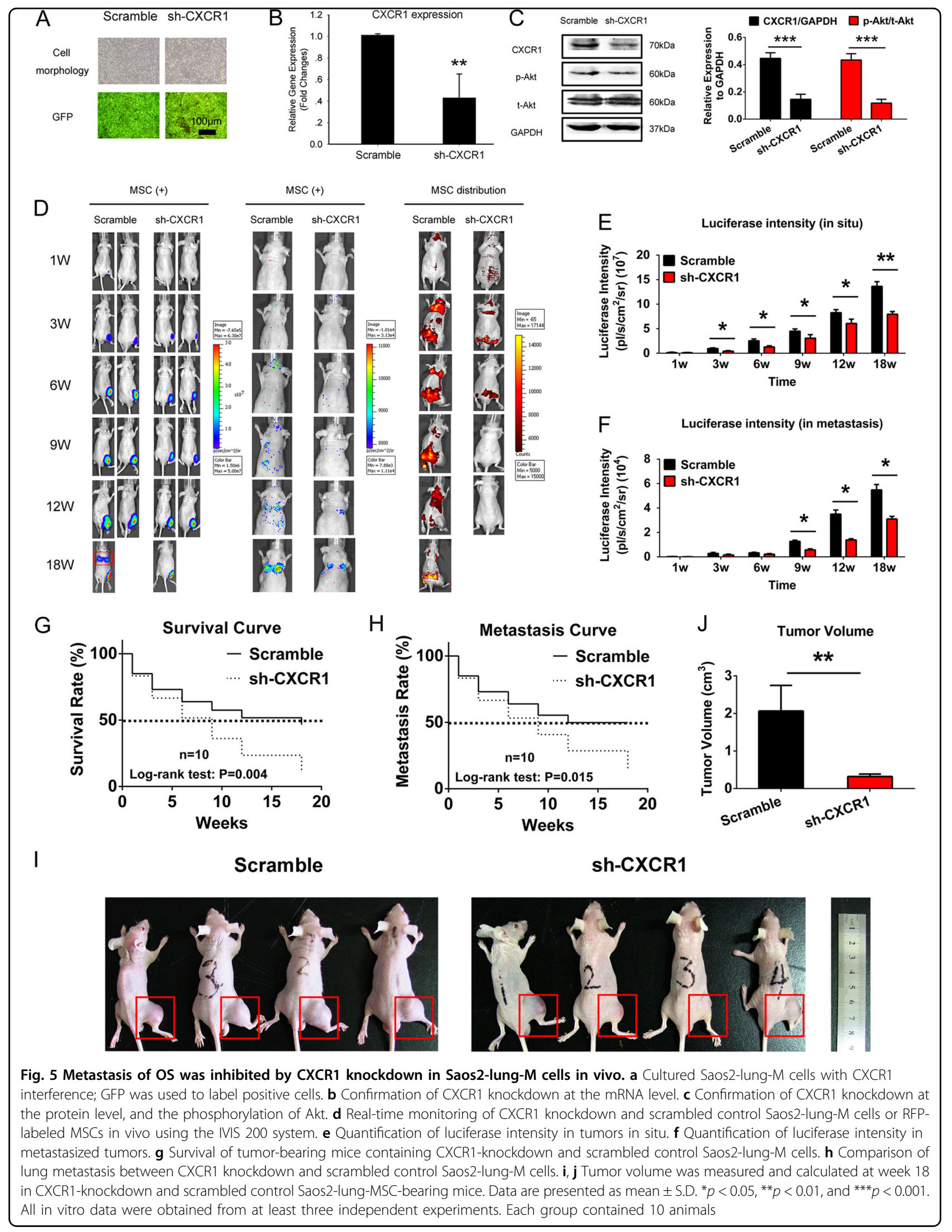




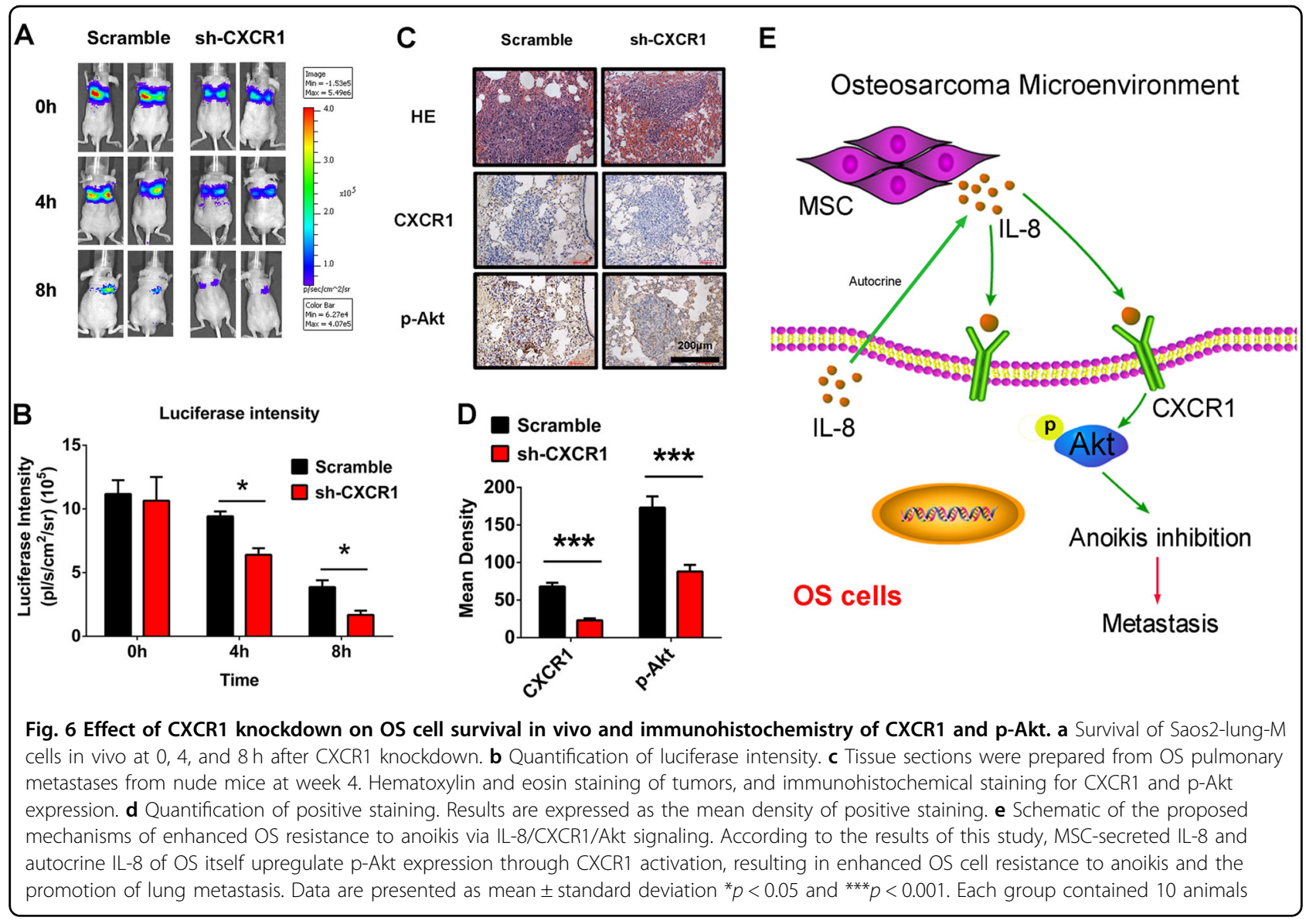

protein and uncovered the IL-8/CXCR1/Akt pathway ${ }^{34}$. Based on this background, we focused on the potential effect of IL-8 on OS cell anoikis, revealing that IL-8 could inhibit OS cell anoikis by activating Akt. Furthermore, MSC-CM treated with IL-8-neutralizing antibody also inhibited OS cell anoikis. However, the influence of IL-8neutralizing antibody treatment of MSC-CM was stronger than that in a medium containing IL-8. Therefore, there may be active components secreted by MSCs that help OS cells escape from anoikis in addition to IL-8. Our previous study showed that MSCs in the bone microenvironment promoted OS progression and protected tumor cells from drug-induced apoptosis through IL-6/ STAT3 signaling ${ }^{35}$.

Although the malignancy of both Saos2-lung-MSC and Saos2-lung cells was significantly higher than that detected in the Saos2-luc parental cells, the metastatic potential of Saos2-lung-M cells was significantly higher than that of Saos2-lung cells. This suggests that MSCs play a role as "selectors" in the process of OS metastasis, perhaps by secreting IL-8 to upregulate the expression of the CXCR1/ Akt signal pathway components in OS cells (Fig. 6e). Another potential mechanism involves autocrine effects, which have been shown to be important in the self- regulation of tumor cells ${ }^{36}$. We also found that IL-8 production was highly upregulated in both metastatic Saos2-lung-M and Saos2-lung cells.

In summary, the present study showed that MSCs enhanced OS cell resistance to anoikis, survival, and metastasis in an IL-8/CXCR1/Akt pathway-regulated manner. By blocking IL-8/CXCR1/Akt signaling via CXCR1 knockdown in tumor cells, the pulmonary metastasis of OS was inhibited and the survival of OSbearing nude mice was prolonged. These results indicate that CXCR1 is a key target in the regulation of the pulmonary metastasis of OS cells.

\section{Materials and methods Main materials}

A human IL-8 ELISA kit was purchased from R\&D systems (Minneapolis, MN, USA, S8000C). Antibodies against p-Akt (4060S), t-Akt (4685S), and GAPDH (2118S), and secondary antibodies (5151S) were purchased from Cell Signaling Technology, Inc. (CST; Danvers, MA, USA). Antibodies against CXCR1 were purchased from Sigma (Darmstadt, Germany, SAB2700216). IL-8 was purchased from PeproTech., Inc. (Rocky Hill, NJ, USA). 


\section{OS cell culture}

Saos2 cells were purchased from the Chinese Academy of Sciences (Shanghai, China). This cell line was identified by the Genetic Testing Biotechnology Corporation (Suzhou, China) using short tandem repeat markers. Saos2-luc cells are luciferase-labeled Saos2 cells (Saos2-luc), which were described in our previous report ${ }^{26}$. The Saos2-lung cell line, which is derived from the Saos2-luc line, is a highly metastatic and chemoresistant human OS cell line ${ }^{24,} 25$. All cells were grown in Dulbecco's modified Eagle's medium (DMEM; Hyclone, Tauranga, New Zealand) supplemented with 10\% FBS (Hyclone), $100 \mathrm{U} / \mathrm{mL}$ penicillin, and $100 \mu \mathrm{g} / \mathrm{mL}$ streptomycin in a $5 \% \mathrm{CO}_{2}$ humidified atmosphere at $37^{\circ} \mathrm{C}$.

\section{MSC culture and preparation of MSC-CM}

MSCs were obtained from proximal femurs during orthopedic surgery as previously described ${ }^{35}$ in accordance with the ethical guidelines of the Shanghai Ninth People's Hospital, Shanghai, China. To prepare the MSC$\mathrm{CM}$, MSCs were grown to $80 \%$ confluence in $10-\mathrm{cm}$ dishes in $\alpha$-minimal essential medium in a $5 \% \mathrm{CO}_{2}$ humidified atmosphere at $37^{\circ} \mathrm{C}$. The medium was discarded and the cells were further cultured in DMEM containing 5\% FBS for $24 \mathrm{~h}$. The medium was then collected, centrifuged at $1000 \times g$ for $10 \mathrm{~min}$, and passed through $0.22-\mu \mathrm{m}$ filters (Millipore, Billerica, MA, USA). All cells were used within five passages.

In addition, we transfected a lentiviral vector system carrying the RFP gene into MSCs to facilitate imaging of MSCs in vivo.

\section{Isolation of highly metastatic Saos2-lung-M cells}

Four-week-old male BALB/c nude mice were injected with $1 \times 10^{7}$ Saos2-luc cells in the right proximal tibia and with $1 \times 10^{7}$ RFP-labeled MSCs via the tail vein. Nude mice with pulmonary metastasis detectable by an IVIS (PerkinElmer, Waltham, MA, USA) were sacrificed when moribund. The lungs were then perfused with phosphatebuffered saline (PBS), excised, finely minced, and incubated for $1 \mathrm{~h}$ in DMEM with $150 \mathrm{IU} / \mathrm{mL}$ collagenase type IV (Sigma-Aldrich, St. Louis, MO, USA). Single-cell suspensions were prepared by repeatedly aspirating the mixture through a 10-mL syringe and then filtering through a nylon mesh. The cells were then pelleted at $1000 \mathrm{rpm}$, washed in PBS, and plated on 6-cm tissue-culture plates in complete medium with $200 \mu \mathrm{g} / \mathrm{mL} \mathrm{G418} \mathrm{(ref.}{ }^{26}$ ) to select for OS cells. The OS cells were allowed to outgrow and form colonies for a few weeks and were then passaged 40 times. The resulting passaged OS cells were named Saos2-lung-M cells.

\section{Analysis of anoikis in vitro}

OS cells were grown to confluence in 10-cm tissue-culture dishes unless otherwise indicated. Trypsinized cells $\left(3 \times 10^{6}\right)$ were quantified and plated on $10-\mathrm{cm}$ dishes coated with poly-hydroxyethylmethacrylate (HEMA), which facilitated maintenance of cells in a suspended state $^{37}$. Poly-HEMA plates were made by adding $4 \mathrm{~mL}$ of a $10 \mathrm{mg} / \mathrm{mL}$ solution of poly-HEMA (Aldrich Chemical Co., Milwaukee, WI, USA) in ethanol to dishes and then drying the dishes in a tissue-culture hood. This coating and drying was repeated, followed by extensive PBS washes. After $24 \mathrm{~h}$, the cells were collected from the poly-HEMA dishes. Anoikis was measured by flow cytometry using Annexin V/propidium iodide double-immunofluorescent staining. Apoptotic events were analyzed as previously described $^{25,38}$.

\section{Analysis of OS cell survival in vivo}

Four-week-old male BALB/c nude mice were injected via the tail vein with $1 \times 10^{7}$ Saos2-luc cells treated with $5 \% \mathrm{FBS}$ or MSC-CM. Surviving OS cells in vivo were detected by bioluminescence at 0,4 , and $8 \mathrm{~h}$. In addition, nude mice were injected via the tail vein with CXCR1knockdown or scrambled Saos2-lung-M cells. OS cell survival in vivo was also detected by bioluminescence at 0 , 4 , and $8 \mathrm{~h}$.

\section{Colony formation assay}

Three six-well plates were seeded with Saos2 cells at a concentration of 600 cells per well in $2 \mathrm{~mL}$ media. After overnight incubation, each well was treated with $5 \%$ bovine serum albumin, 5\% FBS, or MSC-CM. After a 14-day incubation, the media were aspirated from each well and the cells were stained with $1 \%$ crystal violet for $30 \mathrm{~min}$. The number of colonies in each well was counted with plates tested in duplicate as described in a previous study ${ }^{39}$.

\section{ELISA}

Saos2, Saos2-lung, and Saos2-lung-M cells were plated in six-well plates at $1 \times 10^{4}$ cells per well. The next day, the medium was replaced with $2 \mathrm{~mL}$ fresh serum-free DMEM per well, and the culture supernatants were collected after $24 \mathrm{~h}$. IL-8 concentrations in the culture supernatants, as well as in the MSC-CM and normal medium, were measured by ELISA according to the manufacturer's protocols (R\&D Systems, Minneapolis, MN, USA).

\section{RNA isolation and real-time PCR}

Total RNA was isolated from Saos2, Saos2-lung, and Saos2-lung-M cells using an RNeasy Mini Kit (Qiagen, Dusseldorf, Germany), and complementary DNA was synthesized using the iScript cDNA Synthesis Kit (BioRad, Hercules, CA, USA). Subsequently, real-time PCR was performed using an ABI 7500 Sequence Detection System (Thermo Scientific, Waltham, MA, USA) and SYBR Premix Ex Taq (Takara, Dalian, Liaoning, China). All procedures were performed according to the 
Table 1 Sequences of primers used in real-time PCR

\begin{tabular}{|c|c|c|}
\hline \multirow{2}{*}{$\begin{array}{l}\text { Gene } \\
\text { CXCR1 }\end{array}$} & \multicolumn{2}{|c|}{ Primer sequences $\left(5^{\prime}-3^{\prime}\right)$} \\
\hline & Forward & CTGAGCCCCAAGTGGAACGAGACA \\
\hline & Reverse & GCACGGAACAGAAGCTITATTAGGA \\
\hline \multirow[t]{2}{*}{ CXCR2 } & Forward & CAATGAATGAATGAATGGCTAAG \\
\hline & Reverse & AAAGTITCAAGGTTCGTCCGTGTT \\
\hline \multirow[t]{2}{*}{ CXCR3 } & Forward & CCCGCAACTGGTGCCGAGAAAG \\
\hline & Reverse & AGGCGCAAGAGCAGCATCCACAT \\
\hline \multirow[t]{2}{*}{ CXCR4 } & Forward & ATCCCTGCCCTCCTGCTGACTATTC \\
\hline & Reverse & GAGGGCCTTGCGCTTCTGGTG \\
\hline \multirow[t]{2}{*}{ CXCR5 } & Forward & ТССССТССТСАСТСССТTСССАТАA \\
\hline & Reverse & CCTGCGGTTCCATCTGAGTGACATC \\
\hline \multirow[t]{2}{*}{ CXCR6 } & Forward & TTGTTAATAGCTTGCGCATTCTCAT \\
\hline & Reverse & ATCCCCCTTGGTTTCAGCATTCTT \\
\hline \multirow[t]{2}{*}{ CX3CR } & Forward & ATAGATTCCCCATTGCCTCCTC \\
\hline & Reverse & GGTITTCTATTTCCCTTACTGG \\
\hline \multirow[t]{2}{*}{$\beta$-actin } & Forward & CCAACCGCGAGAAGATGA \\
\hline & Reverse & CCAGAGGCGTACAGGGATAG \\
\hline
\end{tabular}

manufacturer's protocols and a previous study ${ }^{40}$. Primer sequences are listed in Table 1.

\section{Western blot}

Whole-cell extracts were prepared by lysing cells in RIPA buffer [150 mM NaCl, 1\% sodium deoxycholate, $0.1 \%$ sodium dodecyl sulfate (SDS), $50 \mathrm{mM}$ Tris- $\mathrm{HCl} \mathrm{pH}$ 7.4, $1 \mathrm{mM}$ EDTA, $1 \mathrm{mM}$ PMSF, and 1\% Triton X-100] containing a cocktail of protease and phosphatase inhibitors. Equal amounts of each protein sample $(30-50 \mu \mathrm{g})$ were separated by SDS-polyacrylamide gel electrophoresis (PAGE) and transferred to polyvinylidene fluoride membranes. The membranes were probed with primary antibodies against CXCR1, p-Akt, t-Akt, and GAPDH. The target proteins were detected using the Odyssey Infrared Imaging System (LI-COR Biosciences, Lincoln, NE, USA).

\section{Immunofluorescence staining}

In brief, the cells were fixed with $4 \%$ paraformaldehyde, stained with rhodamine-linked CXCR1 primary antibody and Alexa 488-linked p-Akt primary antibody, and then counterstained with DAPI. Images of cells were captured using a confocal microscope (LSM510; Carl Zeiss).

\section{CXCR1 knockdown in Saos2-lung-M cells}

Saos2-lung- $M$ cells were transfected with lentiviral particles loaded with either small hairpin (sh)-RNA targeting CXCR1 (5'-TTCTAGGGATGCTGATGCT-3') or a scrambled control (Thermo Fisher Scientific, Waltham, MA, USA) as described previously ${ }^{25}$.

\section{Animal models}

Four-week-old nude mice (BALB/c, nu/nu; SIPPR-BK Laboratory Animal Co. Ltd, Shanghai, China) were housed under pathogen-free conditions at $26-28{ }^{\circ} \mathrm{C}$ with 50-65\% humidity. All animal operations were approved by the Animal Ethics Committee of Shanghai Ninth People's Hospital, Shanghai Jiao Tong University School of Medicine (A-2016-017). For intratibial injections, CXCR1-knockdown or scrambled control Saos2-lung-M cells were harvested, counted, and resuspended in PBS to a final concentration of $2 \times 10^{7}$ cells $/ \mathrm{mL}$. In addition, RFPlabeled MSCs were harvested, counted, and resuspended in PBS to a final concentration of $4 \times 10^{7}$ cells $/ \mathrm{mL}$. Trypan blue exclusion determined that $>95 \%$ cells were viable prior to injection. The animals were anesthetized with $3.5 \%$ pentobarbital, and then $1 \times 10^{6}$ OS cells and $2 \times 10^{6}$ MSCs cells in $50 \mu \mathrm{L}$ of PBS were injected into the proximal tibia using a 25-gauge needle. The tumor volumes were calculated using the formula: volume $=0.2618 \times L \times$ $W \times(L+W)$, where $W$ and $L$ represent the average width and length of the tumor, respectively ${ }^{25}$.

\section{Immunohistochemistry}

The mice were sacrificed and tumor samples from each nude mouse were fixed in $4 \%$ paraformaldehyde, and then immunohistochemically stained for CXCR1 and p-Akt as described previously ${ }^{41}$.

\section{Statistical analysis}

Statistical analyses were performed using SPSS software version 15.0 (SPSS Inc., Chicago, IL, USA). Data are presented as mean \pm standard deviation. Comparisons between two groups were performed using Student's $t$ test, while one-way analysis of variance was used for multiple comparisons. Analysis of survival and metastasis was performed using log-rank tests. Each sample was analyzed in triplicate and experiments were repeated thrice. $p<0.05$ indicates a statistically significant difference.

\section{Acknowledgements}

This work was supported by the National Natural Science Foundation of China (Grant numbers: 81502326 and 81672205) and the Innovation Program for PhD students in Shanghai Jiao Tong University School of Medicine (BXJ201829) and Open Project of Shanghai Key Laboratory of Orthopedic Implants (KFKT2017002).

\section{Authors' contributions}

L.D. and X.-g.H. conducted the experiments and L.D., X.-g.H., B.T., M.-q.W., H.Q, and S.-h.Z. were involved in data analysis. X.-g.H. helped draft the manuscript. T.-t.T. and Q.-m.F. designed the study, analyzed and interpreted the data, and drafted the manuscript. All authors read and approved the final manuscript. Please change Shanghai Jiaotong University School of Medicine to Shanghai Jiao Tong University School of Medicine in Author details section 


\section{Author details}

'Shanghai Key Laboratory of Orthopedic Implants, Department of Orthopedic Surgery, Shanghai Ninth People's Hospital, Shanghai Jiao Tong University School of Medicine, Shanghai, China. 'Department of Orthopaedic Surgery, Renji Hospital, Shanghai Jiao Tong University School of Medicine, Shanghai, China. ${ }^{3}$ Department of Orthopaedic Surgery, Shanghai Sixth People's Hospital Affiliated to Shanghai Jiao Tong University, Shanghai, China

\section{Conflict of interest}

The authors declare that they have no conflict of interest.

\section{Publisher's note}

Springer Nature remains neutral with regard to jurisdictional claims in published maps and institutional affiliations.

Received: 24 March 2018 Revised: 23 April 2018 Accepted: 29 May 2018 Published online: 18 June 2018

\section{References}

1. Lettieri, C. K. et al. Progress and opportunities for immune therapeutics in osteosarcoma. Immunotherapy 8, 1233-1244 (2016).

2. Ottaviani, G. \& Jaffe, N. The epidemiology of osteosarcoma. Cancer Treat. Res. 152, 3-13 (2009).

3. Chen, D. et al. Super enhancer inhibitors suppress MYC driven transcriptional amplification and tumor progression in osteosarcoma. Bone Res. 6, 11 (2018).

4. Jones, K. B. Osteosarcomagenesis: modeling cancer initiation in the mouse. Sarcoma 2011, 694136 (2011).

5. Shaikh, A. B. et al. Present advances and future perspectives of molecular targeted therapy for osteosarcoma. Int. J. Mol. Sci. 17, 506 (2016).

6. Lewis, I. J. et al. Improvement in histologic response but not survival in osteosarcoma patients treated with intensified chemotherapy: a randomized phase III trial of the European Osteosarcoma Intergroup. J. Natl. Cancer Inst. 99, 112-128 (2007).

7. Wu, P. K. et al. Primary osteogenic sarcoma with pulmonary metastasis: clinical results and prognostic factors in 91 patients. Jpn J. Clin. Oncol. 39, 514-522 (2009).

8. Yan, G. N., Lv, Y. F. \& Guo, Q. N. Advances in osteosarcoma stem cell research and opportunities for novel therapeutic targets. Cancer Lett. 370, 268-274 (2016).

9. Simpson, C. D., Anyiwe, K. \& Schimmer, A. D. Anoikis resistance and tumor metastasis. Cancer Lett. 272, 177-185 (2008).

10. Qiao, H. \& Tang, T. Engineering 3D approaches to model the dynamic microenvironments of cancer bone metastasis. Bone Res. 6, 3 (2018).

11. Diaz-Montero, C. M., Wygant, J. N. \& Mclntyre, B. W. PI3-KJAkt-mediated anoikis resistance of human osteosarcoma cells requires Src activation. Eur. J. Cancer 42, 1491-1500 (2006).

12. Cortini, M., Avnet, S. \& Baldini, N. Mesenchymal stroma: role in osteosarcoma progression. Cancer Lett. 405, 90-99 (2017).

13. Mutsaers, A. J. \& Walkley, C. R. Cells of origin in osteosarcoma: mesenchymal stem cells or osteoblast committed cells? Bone 62, 56-63 (2014).

14. Tewari, D. et al. Ovariectomized rats with established osteopenia have diminished mesenchymal stem cells in the bone marrow and impaired homing, osteoinduction and bone regeneration at the fracture site. Stem Cell Rev. 11, 309-321 (2015).

15. Tsukamoto, S. et al. Mesenchymal stem cells promote tumor engraftment and metastatic colonization in rat osteosarcoma model. Int. J. Oncol. 40, 163-169 (2012).

16. Bian, Z. Y., Fan, Q. M., Li, G., Xu, W. T. \& Tang, T. T. Human mesenchymal stem cells promote growth of osteosarcoma: involvement of interleukin- 6 in the interaction between human mesenchymal stem cells and Saos-2. Cancer Sci. 101, 2554-2560 (2010).

17. Xu, W. T., Bian, Z. Y., Fan, Q. M., Li, G. \& Tang, T. T. Human mesenchymal stem cells (hMSCs) target osteosarcoma and promote its growth and pulmonary metastasis. Cancer Lett. 281, 32-41 (2009).

18. Uccelli, A., Moretta, L. \& Pistoia, V. Mesenchymal stem cells in health and disease. Nat. Rev. Immunol. 8, 726-736 (2008).

19. Barcellos-de-Souza, P., Gori, V., Bambi, F. \& Chiarugi, P. Tumor microenvironment: bone marrow-mesenchymal stem cells as key players. Biochim. Biophys. Acta 1836, 321-335 (2013).

20. Gales, D., Clark, C., Manne, U. \& Samuel, T. The chemokine CXCL8 in carcinogenesis and drug response. ISRN Oncol. 2013, 859154 (2013).

21. Abdul-Aziz, A. M. et al. MIF-induced stromal PKCbeta/IL8 is essential in human acute myeloid leukemia. Cancer Res. 77, 303-311 (2017).

22. Avnet, S. et al. Cancer-associated mesenchymal stroma fosters the stemness of osteosarcoma cells in response to intratumoral acidosis via NF-kappaB activation. Int. J. Cancer 140, 1331-1345 (2017).

23. Jiang, H., Wang, X., Miao, W., Wang, B. \& Qiu, Y. CXCL8 promotes the invasion of human osteosarcoma cells by regulation of PI3K/Akt signaling pathway. APMIS 125, 773-780 (2017).

24. Du, L., Fan, Q., Tu, B., Yan, W. \& Tang, T. Establishment and characterization of a new highly metastatic human osteosarcoma cell line derived from Saos2. Int. J. Clin. Exp. Pathol. 7, 2871-2882 (2014).

25. Han, X. G. et al. CXCR1 knockdown improves the sensitivity of osteosarcoma to cisplatin. Cancer Lett. 369, 405-415 (2015).

26. Du, L. et al. Tumorigenesis and spontaneous metastasis by luciferase-labeled human xenograft osteosarcoma cells in nude mice. Chin. Med. J. (Engl.). 125 4022-4030 (2012).

27. Gorlick, R. \& Khanna, C. Osteosarcoma. J. Bone Miner. Res. 25, 683-691 (2010)

28. Hall, B., Andreeff, M., \& Marini, F. The participation of mesenchymal stem cells in tumor stroma formation and their application as targeted-gene delivery vehicles. Handb. Exp. Pharmacol. 180, 263-283 (2007).

29. Mi, F. \& Gong, L. Secretion of interleukin- 6 by bone marrow mesenchymal stem cells promotes metastasis in hepatocellular carcinoma. Biosci. Rep. 37, 4 (2017).

30. Chow, M. T. \& Luster, A. D. Chemokines in cancer. Cancer Immunol. Res. 2, 1125-1131 (2014).

31. Liu, Q. et al. The CXCL8-CXCR1/2 pathways in cancer. Cytokine Growth Factor Rev. 31, 61-71 (2016).

32. Carnero, A. The PKB/AKT pathway in cancer. Curr. Pharm. Des. 16, 34-44 (2010).

33. Engelman, J. A. Targeting PI3K signalling in cancer: opportunities, challenges and limitations. Nat. Rev. Cancer 9, 550-562 (2009).

34. Waugh, D. J. \& Wilson, C. The interleukin-8 pathway in cancer. Clin. Cancer Res. 14, 6735-6741 (2008)

35. Tu, B., Du, L., Fan, Q. M., Tang, Z. \& Tang, T. T. STAT3 activation by IL-6 from mesenchymal stem cells promotes the proliferation and metastasis of osteosarcoma. Cancer Lett. 325, 80-88 (2012).

36. Hiratsuka, S. et al. The S100A8-serum amyloid A3-TLR4 paracrine cascade establishes a pre-metastatic phase. Nat. Cell Biol. 10, 1349-1355 (2008).

37. Folkman, J. \& Moscona, A. Role of cell shape in growth control. Nature $\mathbf{2 7 3}$ 345-349 (1978).

38. Frisch, S. M. \& Francis, H. Disruption of epithelial cell-matrix interactions induces apoptosis. J. Cell Biol. 124, 619-626 (1994).

39. $\mathrm{Hu}, \mathrm{C}$. et al. Antitumor effect of focal adhesion kinase inhibitor PF562271 against human osteosarcoma in vitro and in vivo. Cancer Sci. 108, 1347-1356 (2017).

40. Wang, T. et al. CHIP regulates bone mass by targeting multiple TRAF family members in bone marrow stromal cells. Bone Res. 6, 10 (2018).

41. Tu, B. et al. Osteosarcoma cells promote the production of pro-tumor cytokines in mesenchymal stem cells by inhibiting their osteogenic differentiation through the TGF-beta/Smad2/3 pathway. Exp. Cell Res. 320, 164-173 (2014). 